\title{
Mannose Impairs Lung Adenocarcinoma Growth and Enhances the Sensitivity of A549 Cells to Carboplatin
}

This article was published in the following Dove Press journal: Cancer Management and Research

Introduction: Mannose, a major monosaccharide component of $\mathrm{N}$-glycans, involves in the glycometabolism of human body. Recently, mannose has been shown to suppress tumor growth through enhancing chemosensitivity and reducing the activity of mannose phosphate isomerase (MPI). However, it is largely unknown whether mannose exerts effects on nonsmall cell lung cancer (NSCLC).

Materials and Methods: First, a mannose IC50 assay was conducted to find a suitable concentration of mannose for cell experiments. Then, vitro studies including CCK-8 assay, scratch wound healing assay, and TUNEL assay were performed to evaluate the effects of mannose on A549 cells, and an animal model was established to evaluate the antitumoural effect of mannose on NSCLC in vivo. Finally, immunohistochemistry was done to detect the expression of MPI by Rabbit Anti-MPI.

Results: In this study, a concentration of mannose, $15 \mathrm{mM}$, was used to explore the suppressive effect of mannose on A549 cells. CCK-8 assay demonstrated that mannose significantly inhibited the proliferation of A549 cells and enhanced the anti-tumor efficacy of carboplatin. Wound healing assay showed that mannose inhibits the migration of A549 cells, and mannose-induced migration inhibition was more efficient in A549 cells treated with carboplatin. TUNEL assay demonstrated that mannose significantly enhanced the efficacy of carboplatin to promote apoptosis treated by mannose $(15 \mathrm{mM})$ or carboplatin. The results of animal experiments revealed that the size and weight of tumors derived from A549 cells treated with mannose were smaller than those derived from control cells, and cotreatment with mannose and carboplatin had most efficient inhibition on tumor growth. MPI expression detection showed that the expression level of MPI in the stage Tis (tumor in situ) was the highest, while the stage IV has the lowest.

Discussion: Collectively, our findings suggest that mannose inhibited cell proliferation and migration, promoted cell apoptosis and enhanced the efficacy of carboplatin in lung adenocarcinoma. Preliminary results showed that mannose had less side effect on health. In the future, mannose may be a potential candidate drug for adjuvant therapy of lung adenocarcinoma.

Keywords: mannose, NSCLC, cancer

\section{Introduction}

Lung cancer is one of the most prevalent cancers and the leading causes of cancerrelated death worldwide. ${ }^{1}$ According to the latest Chinese lung cancer report, there were 781,500 new diagnosed cases along with 626,400 of the lung cancer-related death in $2014 .^{2}$ Approximately $85 \%$ of lung cancer cases are non-small cell lung
Department of Respiratory Medicine,

Zhongda Hospital, School of Medicine,

Southeast University, Dingjiaqiao 87,

Gulou District, Nanjing 210009, Jiangsu,

People's Republic of China

Email hanshuhua0922@I26.com
Cancer Management and Research 2020:12 I I077-I I 083

I 1077 
cancer (NSCLC), and lung adenocarcinoma is the commonest subtype of NSCLC. ${ }^{2}$ Majority of lung cancer patients are diagnosed at advanced stages. Nowadays, a variety of treatment strategies, including chemotherapy, radiotherapy, novel targeted therapy and immunotherapy have been developed to improve the survival of advanced NSCLC. ${ }^{3}$ However, the drug resistance remains the major cause of treatment failure and relapse in NSCLC.

Mannose is a glucose-related simple sugar. Previous studies on infection revealed that mannose and its derivative, mannoside, were beneficial to motivate the adhesion of bacteria in infection. ${ }^{4}$ Nowadays, it was found that mannose could influence tumor growth. Growing tumor cells have increased metabolic demands outstrips energy supply, while the main resource of energy is saccharides. Recently, a research reported that mannose could impair tumor growth and enhance chemotherapy response in solid-tumor xenografted model without obvious side effects. ${ }^{5}$ The anti-tumor effect of mannose was depended on the expression of mannose phosphate isomerase (MPI). As a major enzyme In glucose metabolism, MPI was more effective in tumors with relatively lower MPI expression. ${ }^{6}$ And MPI inhibitor restored the sensitivity of MPI-overexpressed tumors to mannose. ${ }^{5}$ All these previous findings indicated that mannose might be useful in the treatment of human malignances.

In the present study, we aimed to investigate the effect of mannose on lung adenocarcinoma both in vitro and in vivo.

\section{Materials and Methods}

\section{Reagents}

RPMI-1640, fetal bovine serum (FBS) and penicillin/ streptomycin were purchased from Thermo Fisher Scientific, Inc. (Waltham, Ma, USA). Cell counting Kit-8 (CCK-8) was purchased from Dojindo molecular Technologies, Inc. (Kumamoto, Japan). Rabbit Anti-MPI/ Mannose Phosphate Isomerase antibody (bs-18654R) was purchased from Bioss, Inc. (Beijing, China).

\section{Cell Culture}

The human lung adenocarcinoma cell line A549 was purchased from the Cell Bank of the Chinese Academy of Science (Shanghai, China), and cultured in RPMI-1640 medium supplemented with $10 \% \mathrm{FBS}, 1 \times 10 \mu \mathrm{g} / \mathrm{mL}$ penicillin and $1 \times 10 \mu \mathrm{g} / \mathrm{mL}$ streptomycin at $37{ }^{\circ} \mathrm{C}$ under a mixture of $95 \%$ air and $5 \% \mathrm{CO}_{2}$.

\section{IC50 and CCK-8 Assay}

Mannose (purity $>99 \%$; Aladdin, Shanghai, China) was stored in solid form and dissolved in RPMI-1640 to different final concentrations $(4,8,12,16,24,32,40,48 \mathrm{mM})$. A549 cells were plated into 96-well plate, and then incubated with various concentrations of mannose for $24 \mathrm{~h}$ to 48h. Following addition of CCK-8 reagent to each well of plate, and $1 \mathrm{~h}$ incubation at $37{ }^{\circ} \mathrm{C}$, the absorbance at a wavelength of $450 \mathrm{~nm}$ was measured with a microplate reader (Bio-Rad Laboratories, Inc., Hercules, CA, USA). Then $\mathrm{IC}_{50}$ was calculated and the concentration less than $\mathrm{IC}_{50}$ was used for CCK-8 assay. The steps of CCK-8 assay were similar to the above. There were four groups [Control; $15 \mathrm{mM}$ mannose (group M); 8mg/L carboplatin (group C); $15 \mathrm{mM}$ mannose $+8 \mathrm{mg} / \mathrm{L}$ carboplatin (group MC)].

\section{Scratch Wound Healing Assay}

To measure the effect of mannose on cell migration ability, scratch wound healing assay was applied. A linear wound was made by scraping a nonopening pipette across the confluent cell layer, 24h after treatment with mannose $(15 \mathrm{mM})$ or carboplatin $(8 \mathrm{mg} / \mathrm{L})$. There were four groups [group control; $15 \mathrm{mM}$ mannose (group $\mathrm{M}$ ); $8 \mathrm{mg} / \mathrm{L}$ carboplatin (group C); $15 \mathrm{mM}$ mannose $+8 \mathrm{mg} / \mathrm{L}$ carboplatin (group MC)]. Cells were washed twice to remove detached cells and debris. And then, the size of wounds was observed and measured under the microscope at the indicated times. The results were analyzed by IMAGE J software (1.48, National Institutes of Health).

\section{TUNEL Assay}

On reaching $75 \%$ confluence, A549 cells were treated with $15 \mathrm{mM}$ mannose or $8 \mathrm{mg} / \mathrm{L}$ carboplatin for $48 \mathrm{~h}$. Then fixed with $4 \%$ paraformaldehyde and permeabilized with $1 \%$ Triton X-100. DNA fragmentation was determined by TdT-mediated dUTP nick end-labeling (TUNEL) as described by the manufacturer. There were four groups [group control; $15 \mathrm{mM}$ mannose (group $\mathrm{M}$ ); $8 \mathrm{mg} / \mathrm{L}$ carboplatin (group C); $15 \mathrm{mM}$ mannose $+8 \mathrm{mg} / \mathrm{L}$ carboplatin (group MC)]. Then, fluorescent images were taken by an EVOS fluorescent microscope (Nikon Corp., Tokyo, Japan). The results were analyzed by IMAGE J software.

\section{In vivo Experiment}

Six-week old female BALB/c nude mice purchased from KeyGEN BioTECH Corp., Ltd. (Jiangsu, China) was raised in specific pathogen-free environment with 12-hour light/dark 


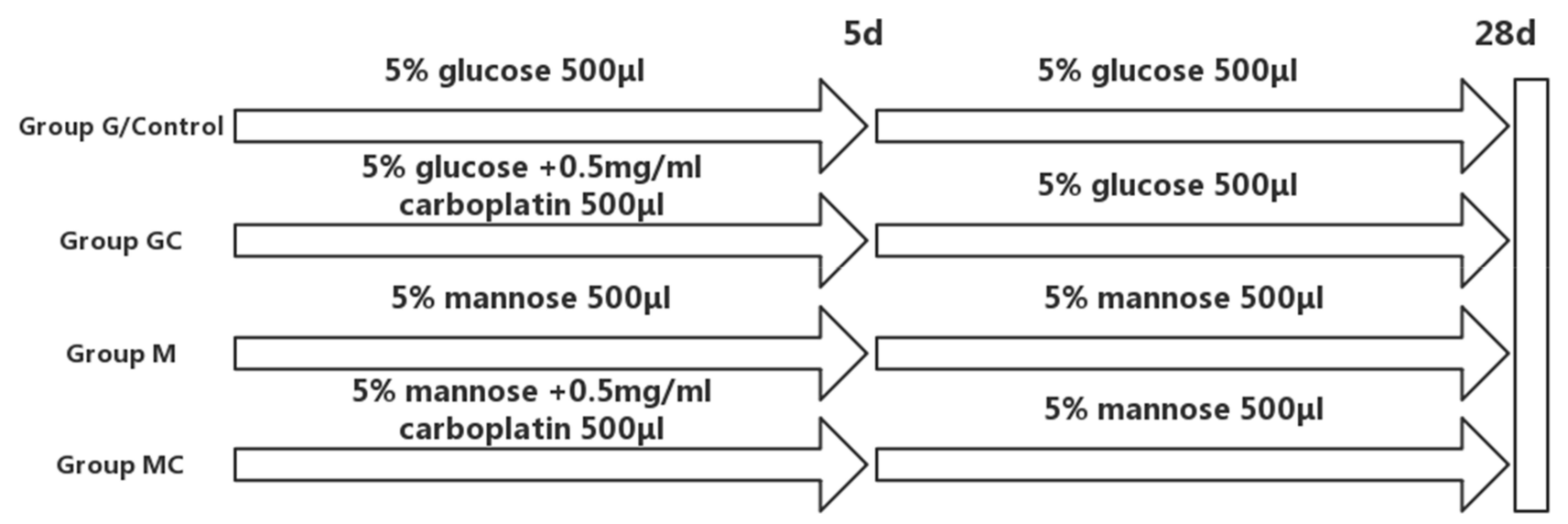

Figure I Detailed schemes of animal experiments.

cycles. A549 cells (1x106) were suspended in $100 \mu \mathrm{L}$ PBS and injected subcutaneously in the left armpit of each mouse. When tumors reached a minimum volume of $1 \mathrm{~cm}^{3}, 16$ Mice were randomly divided into 4 groups of 4 each. The mice were injected intraperitoneally with different reagents [5\% glucose (group G/control); 5\% mannose (group M); 5\% glucose $+0.5 \mathrm{mg} / \mathrm{mL}$ carboplatin (group GC); $5 \%$ mannose $+0.5 \mathrm{mg} / \mathrm{mL}$ carboplatin (group MC)] at 7 p.m. every day. ${ }^{7}$ Detailed scheme is showed in Figure 1. The total injection time for all reagents was $30 \mathrm{~min}$. To evaluate if high-dose exogenous mannose intake would affect the health of mice, we detected the weight and blood glucose of mice. The weight of the mice and the tumor sizes were measured before and 1 day after treatment. And the blood concentration of blood glucose were measured every 14 days. At the 29th day after the first treatment, nude mice were sacrificed by cervical dislocation with no anaesthetic, and tumors were peeled off and weighed. And a portion of each tumor body was made a pathological section for Immunohistochemical tests for the detection of MPI. In the experiment, there were no multiple tumors in nude mice. All protocols were approved by the Animal Ethics Committee of the Medical School, Southeast University (Nanjing, China), and followed the Regulations for Ethical Review of Laboratory Animals in Southeast University for the welfare of the animals. All the experiments were carried on at the Specific Pathogen Free (SPF) Animal House of the Medical School, Southeast University.

\section{MPI Detection}

Each stage (Tis; I; II; III; IV) of lung adenocarcinoma pathological sections and normal lung tissue pathological sections were obtained from Department of pathology of Zhongda Hospital and the quantities of each were about 20
From January 2018 to December 2019. Then immunohistochemistry was done to detect the expression of MPI by Rabbit Anti-MPI. The results of immunohistochemistry were recorded by fluorescence microscope (Nikon Corp., Tokyo, Japan) and analyzed by Image-Pro Plus software (6.0, MEDIA CYBERNETICS).

\section{Statistical Analysis}

All the experiments were performed in triplicate on three independent occasions. All the data are presented as the mean \pm standard deviation of three independent experiments. One-way analysis of variance (one-way ANOVA) were used to analyze the data obtained from multiple groups. Differences were considered statistically significant at $\mathrm{P}<0.05$.

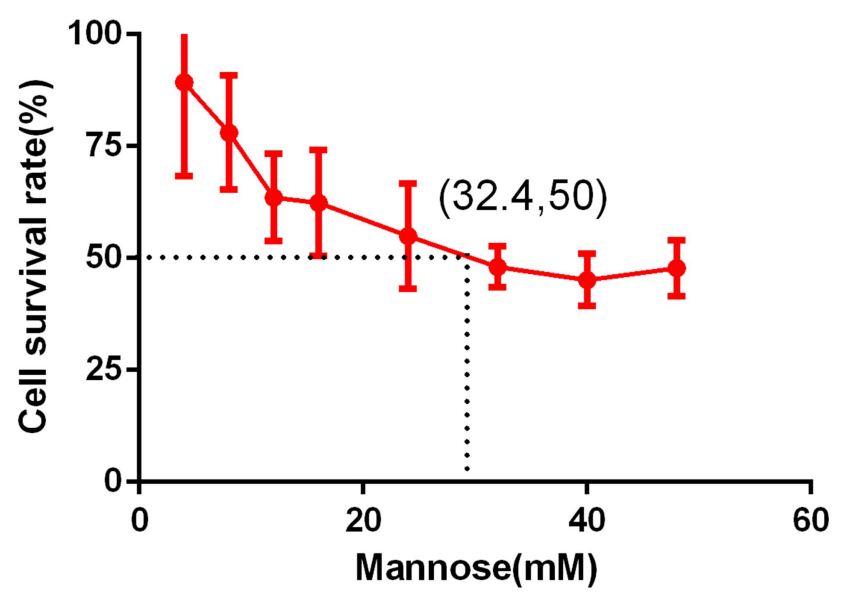

Figure 2 The IC50 assay results of mannose in A549 cells. 


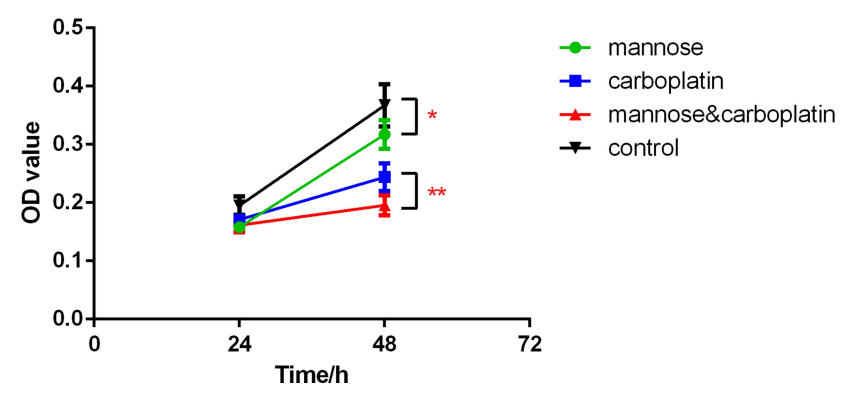

Figure 3 The CCK-8 assay results of mannose in A549 cells. A549 cells were treated with four different intervention conditions for $48 \mathrm{~h}$. The inhibition rate was detected by CCK-8 assay. OD, optical density. Data were presented as the mean \pm standard deviation $(n=3)$; ${ }^{*}<<0.05, * * P<0.01$. CCK-8, Cell Counting Kit-8.

\section{Results}

\section{Mannose Inhibits the Proliferation of} A549 Cells and Enhances the Efficacy of Carboplatin

As shown in Figure 2, the $\mathrm{IC}_{50}$ of mannose was $32.4 \mathrm{mM}$. A lower concentration of mannose, $15 \mathrm{mM}$, was used to explore the suppressive effect of mannose on A549 cells. CCK-8 assay demonstrated that mannose significantly inhibited the proliferation of A549 cells and enhanced the anti-tumor efficacy of carboplatin after $48 \mathrm{~h}$ of treatment (Figure 3).

\section{Mannose Inhibits the Migration in Parental A549 Cells and Carboplatin-Treated A549 Cells}

Wounding healing assay showed that mannose inhibits the migration of A549 cells. More importantly, mannoseinduced migration inhibition was more efficient in A549 cells treated with carboplatin $(8 \mathrm{mg} / \mathrm{L})$ for $24 \mathrm{~h}$ (Figure 4$)$. The results showed that significant differences were observed between 4 groups.

\section{Mannose Enhances the Efficacy of Carboplatin to Promote Apoptosis in A549 Cells}

Results of TUNEL assay demonstrated that mannose significantly enhanced the efficacy of carboplatin to promote apoptosis treated by mannose $(15 \mathrm{mM})$ or carboplatin $(8 \mathrm{mg} / \mathrm{L})$ for $48 \mathrm{~h}$ (Figure 5$)$. The results showed that significant difference was observed between group $\mathrm{C}$ and MC. No difference was observed between group NC (normal control) and group $\mathrm{M}$, but the apoptosis rate of group $\mathrm{M}$ was higher than group control.
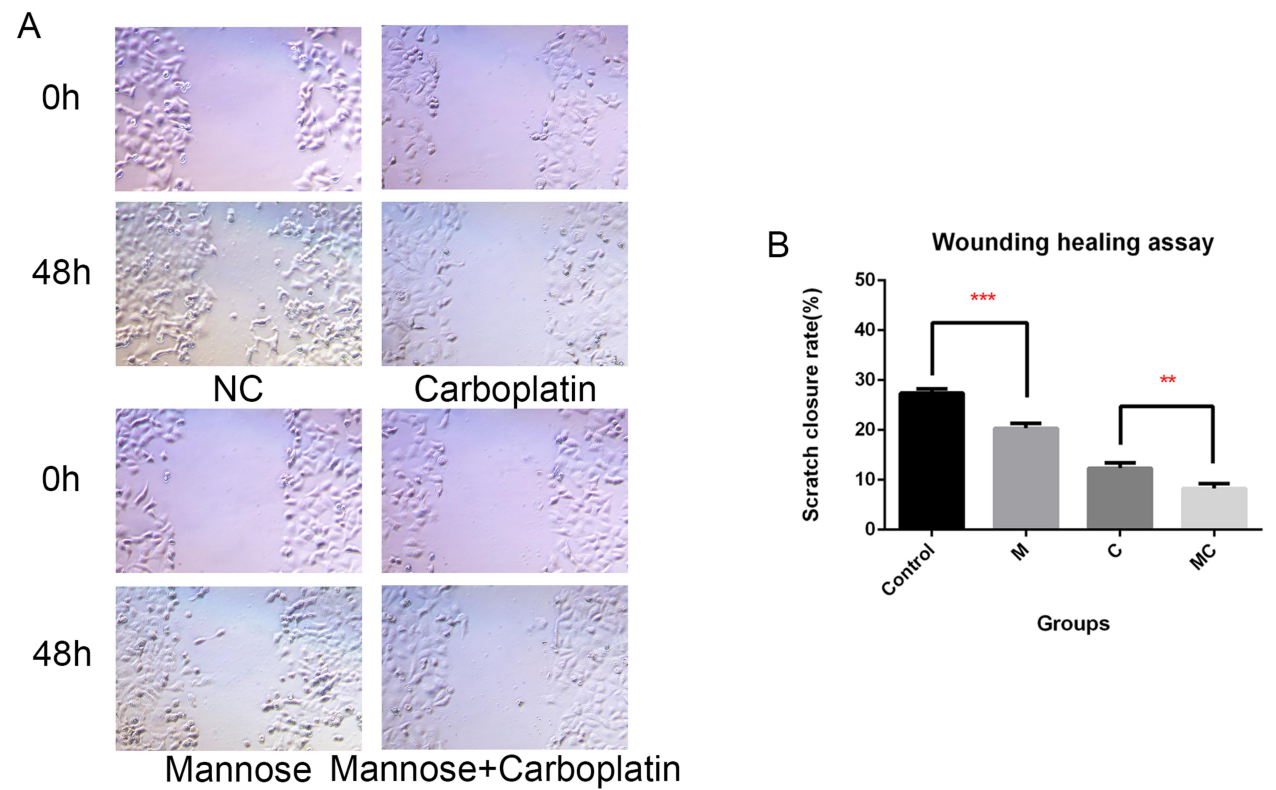

Figure 4 The results of wounding healing assay revealed that mannose in A549 cells. A549 cells treated with four different intervention conditions for $48 \mathrm{~h}$ (A). The scratch closure rate was detected by IMAGE J software (B). Data were presented as the mean \pm standard deviation $(\mathrm{n}=3) ; * * \mathrm{P}<0.0 \mathrm{I}, * * * \mathrm{P}<0.00 \mathrm{I}$.

Abbreviations: M, mannose; C, carboplatin; MC, mannose+carboplatin. 


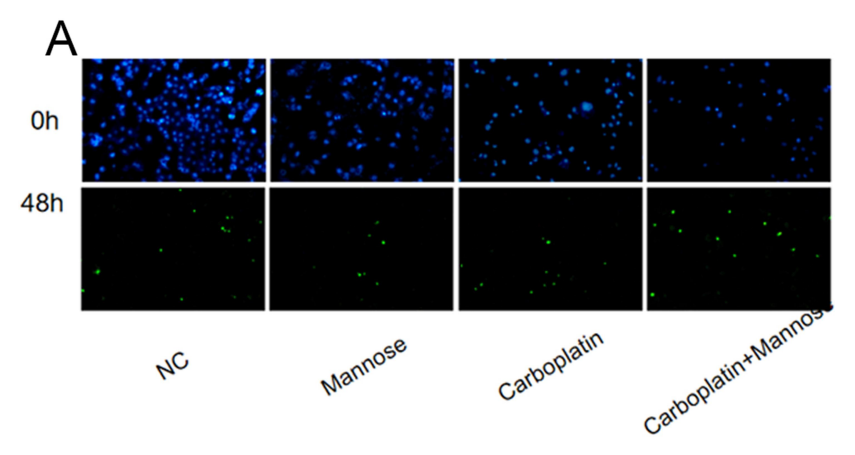

B

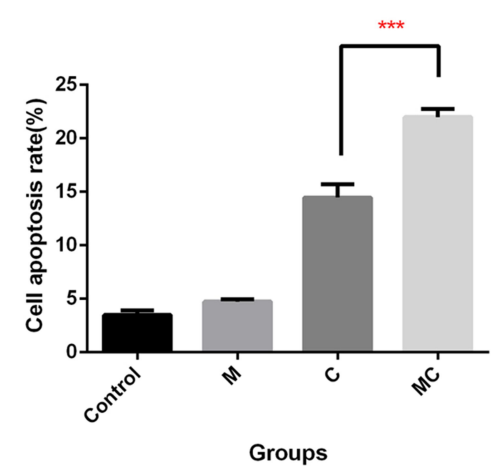

Figure 5 The results of TUNEL assay in A549 cells mannose treated with four different conditions for $48 \mathrm{~h}$ (A, B). TUNEL staining was performed on histological sections of tumor tissue to detect apoptotic cells. Positive TUNEL staining was observed as light green granules in the cytoplasm. Data were presented as the mean \pm standard deviation $(n=3)$; *** ${ }^{*}<0.001$.

Abbreviations: M, mannose; C, carboplatin; MC, mannose+carboplatin; TUNEL, transferase dUTP nick end-labeling.

\section{Mannose Suppresses Tumor Growth in vivo and Enhances the Efficacy of Carboplatin}

Animal experiments were designed to further prove whether mannose inhibited the growth of NSCLC tumor and enhanced the efficacy of carboplatin in vivo. The results revealed that the size and weight of tumors derived from A549 cells treated with mannose was smaller than those derived from control cells. Additionally, co-treatment with mannose and carboplatin had most efficient inhibition on tumor growth (Figure 6). According to ANOVA and Dunnett's test, significant differences were observed between group $\mathrm{M}$ and group $\mathrm{G}$, and significant differences were also observed between group $\mathrm{C}$ and group MC. To monitor the health status of mice, the body weight and blood glucose were detected regularly (Figures 7 and 8). According to ANOVA and Dunnett's test, no difference was found between four groups.

\section{MPI Expression in Lung Adenocarcinoma}

In order to assess MPI expression level and its effect on prognosis, lung adenocarcinoma tissue sections were investigated by immunohistochemistry. The positivity of MPI was found to be negatively correlated with tumor stages. The expression level of MPI in the stage Tis (tumor in situ) was the highest, while the stage IV has the lowest (Figure 9).

\section{Discussion}

Previously, researches have been carried on to study the role of mannose in affecting normal monosaccharide. Decades ago, mannose was used as medicine to treat urinary tract infection. ${ }^{8}$ Recently, a special application of mannose for suppressing tumor growth was found and none of the other monosaccharide had the function of impairing tumor growth. ${ }^{6}$

Mannose has been proven to impair tumor growth and enhance chemotherapy when the expression level and
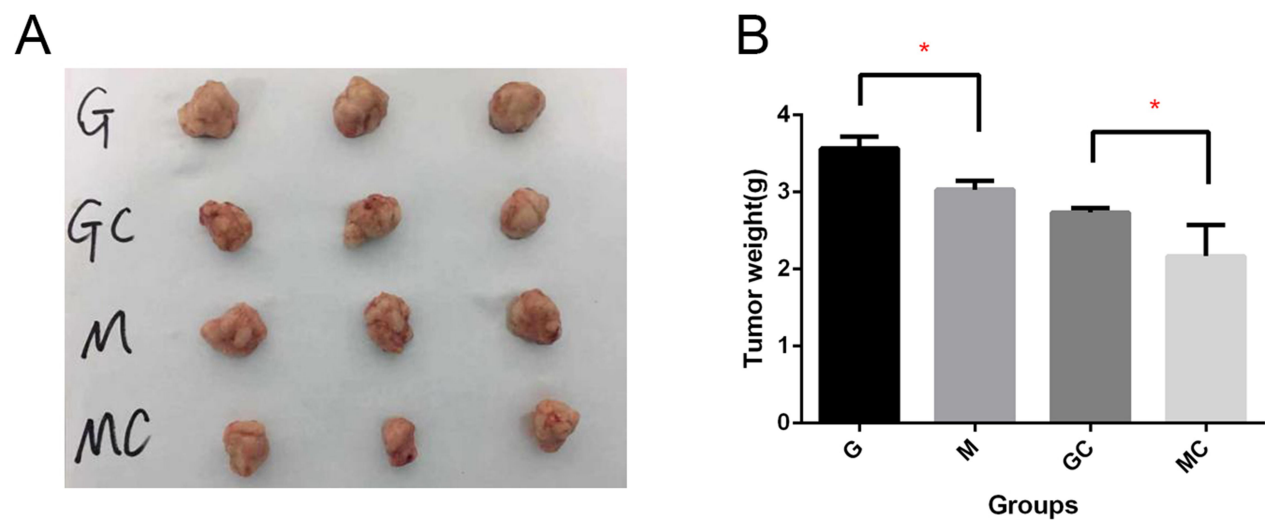

Figure 6 The results of Animal experiments illustrated the effect of mannose on tumor growth. The nude mice that were transplanted with A549 cells were randomly divided into four groups (A). Treatment was administered by intraperitoneal injection of drugs for 28 days. The tumors in each group were dissected and imaged. The measurements of tumor weight were presented $(\mathbf{B})$. Data were presented as the mean \pm standard deviation $(n=3) ;{ }^{*} P<0.05$.

Abbreviations: G, glucose/control; M, mannose; GC, glucose+carboplatin; MC, mannose+carboplatin. 


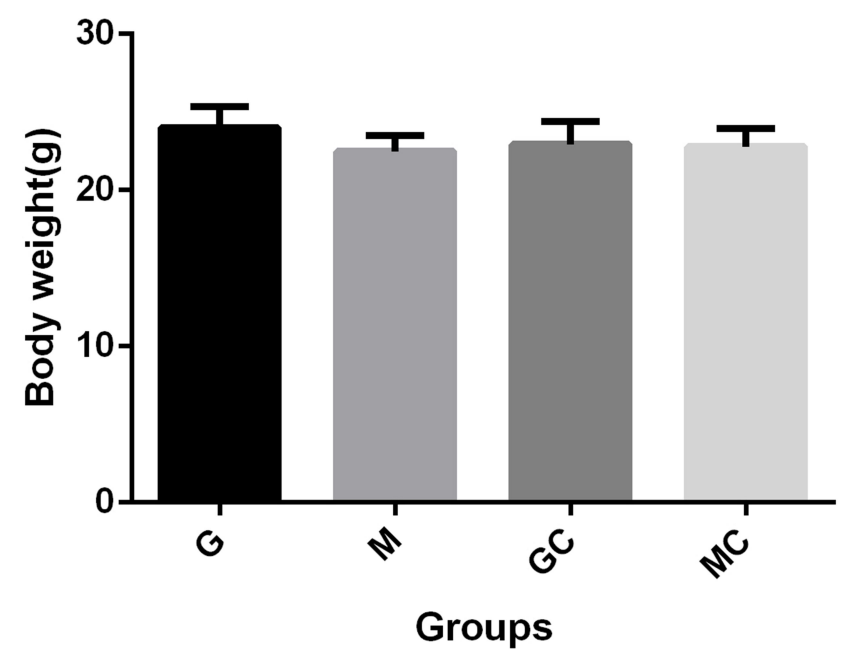

Figure 7 The body weight of mice were measured at 29th day of injection, the mice body weight was measured. Data were presented as the mean \pm standard deviation $(n=3)$.

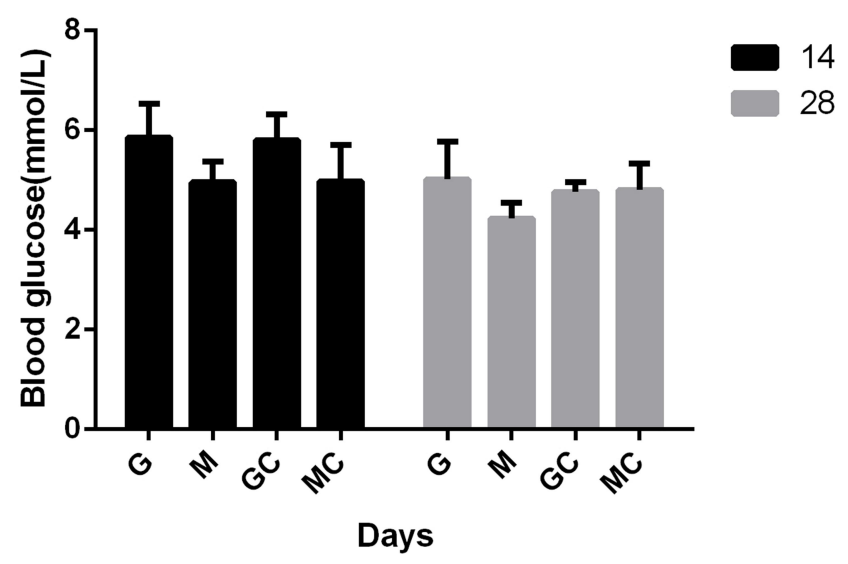

Figure 8 The blood glucose of mice was detected at 29th day of the animal experiment, the mice blood glucose was measured. Data were presented as the mean \pm standard deviation $(n=3)$.

activity of MPI was low among several cancer cells. ${ }^{6}$ According to that experimental results, we were interested in that whether mannose could influence lung cancer cells.
In the present study, our experimental results showed that mannose can inhibit the proliferation and migration of A549 cells and enhance the efficacy of carboplatin in A549-xenografted tumors. Cell apoptosis was significantly increased with the co-treatment of mannose and carboplatin both in vitro and in vivo.

And we evaluated the mice health through detecting the body weight and blood glucose of mice and did not find significant difference between groups. There was no visible influence occurred on mice during experimentation. And the tumor tissue loss had significant difference between groups. Mannose suppressed lung adenocarcinoma cell growth possibly by interfering with glucose metabolism in cancer cells. ${ }^{5,9}$ Researches had proved that the effect of mannose for suppressing cancer was negatively related to the expression level of MPI. ${ }^{5}$ Moreover, the level of MPI was found to be lower in tumor tissues at advanced stage. The expression level of MPI may be connected with the growth and death of cancer cells. As previously reported, MPI loss induced p53 and suppressed the Warburg effect of tumor cells. ${ }^{10}$ Additionally, studies had found that mannose could stimulate Treg cell differentiation in human and mouse cells by promoting TGF- $\beta$ activation in vitro, which might enhance immune function of body. ${ }^{11}$ And the significant association between high level of d-mannose and better prognosis was determined in patients with esophageal adenocarcinoma receiving chemotherapy. $^{12}$ Therefore, increased plasma mannose level maybe a protective factor against cancer.

In conclusion, our findings demonstrated that mannose inhibited cell proliferation and migration, promoted cell apoptosis and enhanced the efficacy of carboplatin in lung adenocarcinoma. Preliminary results showed that mannose had less side effect on health. Therefore, mannose could probably solve the problem of drug resistance during
A

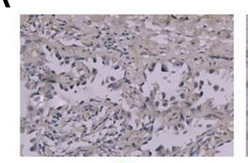

Tis

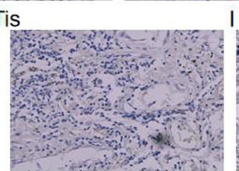

III
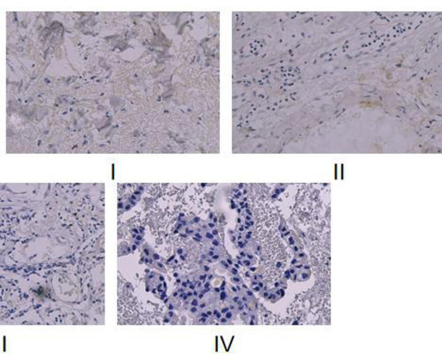

B

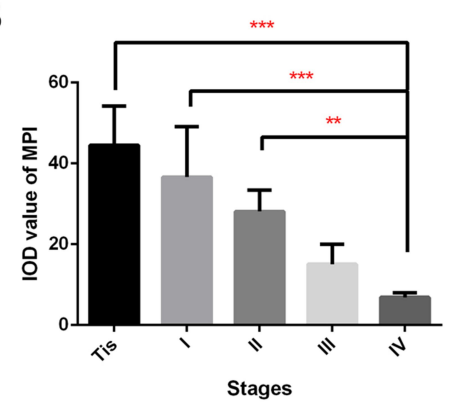

Figure 9 The results of MPI detection of each stage of lung adenocarcinoma tissues. Twenty tissues were collected from lung adenocarcinoma patients in different stage (Tls, I, II, III, IV) and used to detect relative positivity of MPI. The immunohistochemistry results imaged by fluorescence microscope (A). The MPI expression level was detected by Image-Pro Plus software. IOD, integrated optical density (B). Data were presented as the mean \pm standard deviation $(\mathrm{n}=20)$; $* * \mathrm{P}<0.0 \mathrm{I}$, $* * * \mathrm{P}<0.00 \mathrm{I}$. 
chemotherapy. In the future, mannose may be a potential candidate drug for adjuvant therapy of lung adenocarcinoma. The present research had a number of limitations. The specific mechanism through which mannose inhibited cell proliferation and migration, promoted cell apoptosis remain unclear. Much more study will be carried out to explore the mechanism by which mannose and MPI regulated the chemosensitivity in lung adenocarcinoma.

\section{Funding}

No funding was received.

\section{Disclosure}

The authors report no conflicts of interest for this work.

\section{References}

1. Siegel RL, Miller KD, Jemal A, et al. Cancer statistics, 2020. $C A$ Cancer J Clin. 2020;70(1):7-30. doi:10.3322/caac.21590

2. Sun KX, Zheng RS, Zeng HM, et al. The incidence and mortality of lung cancer in China, 2014. Zhonghua Zhong Liu Za Zhi. 2018;40 (11):805-811.

3. Ettinger DS, Wood DE, Aggarwal C, et al. NCCN guidelines insights: non-small cell lung cancer, Version 1.2020. J Natl Compr Canc Netw. 2019;17(12):1464-1472.
4. Caretto M, Giannini A, Russo E, et al. Preventing urinary tract infections after menopause without antibiotics. Maturitas. 2017;99:43-46. doi:10.1016/j.maturitas.2017.02.004

5. Gonzalez PS, O'Prey J, Cardaci S, et al. Mannose impairs tumour growth and enhances chemotherapy. Nature. 2018;563(7733):719723. doi:10.1038/s41586-018-0729-3

6. Sharma V, Ichikawa M, Freeze HH, et al. Mannose metabolism: more than meets the eye. Biochem Biophys Res Commun. 2014;453 (2):220-228. doi:10.1016/j.bbrc.2014.06.021

7. Wojcikowski K, Gobe G. Dose translation from animal to human studies revisited. Phytother Res. 2014;28(1):22-27. doi:10.1002/ ptr.4966

8. Domenici L, Monti M, Bracchi C, et al. D-mannose: a promising support for acute urinary tract infections in women. A Pilot Study. Eur Rev Med Pharmacol Sci. 2016;20(13):2920-2925.

9. DeRossi C, Bode L, Eklund EA, et al. Ablation of mouse phosphomannose isomerase (Mpi) causes mannose 6-phosphate accumulation, toxicity, and embryonic lethality. J Biol Chem. 2006;281 (9):5916-5927. doi:10.1074/jbc.M511982200

10. Shtraizent N, DeRossi C, Nayar S, et al. MPI depletion enhances O-GlcNAcylation of p53 and suppresses the warburg effect. Elife. 2017;6:e22477. doi:10.7554/eLife.22477

11. Zhang D, Chia C, Jiao X, et al. D-mannose induces regulatory T cells and suppresses immunopathology. Nat Med. 2017;23(9):1036-1045. doi: $10.1038 / \mathrm{nm} .4375$

12. Gu J, Liang D, Pierzynski JA, et al. D-mannose: a novel prognostic biomarker for patients with Esophageal adenocarcinoma. Carcinogenesis. 2017;38(2):162-167.

\section{Publish your work in this journal}

Cancer Management and Research is an international, peer-reviewed open access journal focusing on cancer research and the optimal use of preventative and integrated treatment interventions to achieve improved outcomes, enhanced survival and quality of life for the cancer patient.
The manuscript management system is completely online and includes a very quick and fair peer-review system, which is all easy to use. Visit http://www.dovepress.com/testimonials.php to read real quotes from published authors. 